\title{
Are There Differences between Macrocyclic Gadolinium Contrast Agents for Brain Tumor Imaging? Results of a Multicenter Intraindividual Crossover Comparison of Gadobutrol with Gadoteridol (the TRUTH Study)
}

\author{
K.R. Maravilla, M.P. Smith, J. Vymazal, M. Goyal, M. Herman, J.J. Baima, R. Babbel, M. Vaneckova, J. Žižka, C. Colosimo, \\ M. Urbańczyk-Zawadzka, M. Mechl, A.K. Bag, S. Bastianello, E. Bueltmann, T. Hirai, T. Frattini, M.A. Kirchin, and G. Pirovano
}

\begin{abstract}
BACKGROUND AND PURPOSE: Gadobutrol (Gadavist) and gadoteridol (ProHance) have similar macrocyclic molecular structures, but gadobutrol is formulated at a 2-fold higher $(1 \mathrm{~mol} / \mathrm{L}$ versus $0.5 \mathrm{~mol} / \mathrm{L})$ concentration. We sought to determine whether this difference impacts morphologic contrast-enhanced MR imaging.
\end{abstract}

\begin{abstract}
MATERIALS AND METHODS: Two hundred twenty-nine adult patients with suspected or known brain tumors underwent two 1.5T MR imaging examinations with gadoteridol or gadobutrol administered in randomized order at a dose of $0.1 \mathrm{mmol} / \mathrm{kg}$ of body weight. Imaging sequences and $\mathrm{Tl}$ postinjection timing were identical for both examinations. Three blinded readers evaluated images qualitatively and quantitatively for lesion detection and for accuracy in characterization of histologically confirmed brain tumors. Data were analyzed by using the Wilcoxon signed rank test, the McNemar test, and a mixed model.

RESULTS: Two hundred nine patients successfully completed both examinations. No reader noted a significant qualitative or quantitative difference in lesion enhancement, extent, delineation, or internal morphology ( $P$ values $=.69-1.00)$. One hundred thirty-nine patients had at least 1 histologically confirmed brain lesion. Two readers found no difference in the detection of patients with lesions (133/139 versus $135 / 139, P=.317 ; 137 / 139$ versus $136 / 139, P=.564)$, while 1 reader found minimal differences in favor of gadoteridol (136/139 versus 132/139, $P=$.046). Similar findings were noted for the number of lesions detected and characterization of tumors (malignant/benign). Three-reader agreement for characterization was similar for gadobutrol $(66.4 \%[\kappa=0.43])$ versus gadoteridol $(70.3 \%[\kappa=0.45])$. There were no significant differences in the incidence of adverse events $(P=.199)$.
\end{abstract}

CONCLUSIONS: Gadoteridol and gadobutrol at $0.1 \mathrm{mmol} / \mathrm{kg}$ of body weight provide similar information for visualization and diagnosis of brain lesions. The 2-fold higher gadolinium concentration of gadobutrol provides no benefit for routine morphologic imaging.

ABBREVIATIONS: GBCA = gadolinium-based contrast agent; $\mathrm{GRE}=$ gradient recalled-echo; $\mathrm{SE}=$ spin-echo

adobutrol (Gadavist, Gd-BT-DO3A; Bayer HealthCare, Wayne, New Jersey) and gadoteridol (ProHance, Gd-HPDO3A; Bracco Diagnostics, Monroe, New Jersey) are nonionic

Received August 7, 2014; accepted after revision September 15

From the MR Research Laboratory (K.R.M.), University of Washington, Seattle, Washington; Department of Radiology (M.P.S.), Beth Israel Deaconess Medical Center, Boston, Massachusetts; Department of Radiology (J.V.), Na Homolce Hospital, Prague, Czech Republic; Seaman Family MR Research Centre (M.G.), University of Calgary, Calgary, Alberta, Canada; Department of Radiology (M.H.), University Hospital Olomouc, Olomouc, Czech Republic; Clinical Radiologists Service Corporation (J.J.B.), Memorial Medical Center, Springfield, Illinois; Good Samaritan Regional Medical Center (R.B.), Corvallis, Oregon; Department of Magnetic Resonance (M.V.), General University Hospital, Prague, Czech Republic; Department of Diagnostic Radiology (J.Ž.), University Hospital Hradec Králové and Faculty of Medicine in Hradec Králové, Charles University, Prague, Czech Republic; Istituto di Radiologia (C.C.), Policlinico "Agostino Gemelli," Rome, Italy; Department of Radiology (M.U.-Z.), John Paul II Hospital, Krakow, Poland; Department of Radiology (M.M.), University Hospital Brno-Bohunice, Brno, Czech Republic; Department of Radiology (A.K.B.), University of Alabama at Birmingham Medical Center, Birmingham, Alabama; Department of Neuroradiology (S.B), University of Pavia, Pavia, Italy; Institute for Diagnostic and Interventional Neuroradiology (E.B.), Hannover, macrocyclic gadolinium-based contrast agents (GBCAs) approved by the US Food and Drug Administration and other regulatory bodies for MR imaging of the CNS in adults and children older than 2 years of age. ${ }^{1,2}$ The approved routine dose of both agents is $0.1 \mathrm{mmol} / \mathrm{kg}$ of body weight. Structurally, the 2 agents differ only in that a hydroxypropyl group on the gadoteridol molecule is replaced by a trihydroxybutyl group on the gadobutrol molecule. ${ }^{3}$ The published $r 1$ relaxivity values at $1.5 \mathrm{~T}$ are $4.7-5.2$

Germany; Department of Diagnostic Radiology (T.H.), Kumamoto University, Honjo, Kumamoto, Japan; Ospedale Valduce (T.F.), Como, Italy; Global Medical and Regulatory Affairs (M.A.K.), Bracco Imaging SpA, Milan, Italy; and Global Medical and Regulatory Affairs (G.P.), Bracco Diagnostics, Monroe, New Jersey.

Please address correspondence to Kenneth R. Maravilla, MD, Radiology and Neurological Surgery, MR Research Laboratory, University of Washington, 1959 NE Pacific, Box 357115, Seattle, WA 98195; e-mail: kmarav@uw.edu

-- Indicates open access to non-subscribers at www.ajnr.org

Evidence-Based Medicine Level 1.

http://dx.doi.org/10.3174/ajnr.A4154 
$\mathrm{L} \times \mathrm{mmol}^{-1} \times$ second $^{-1}$ for gadobutrol and 4.1-4.3 $\mathrm{L} \times$ mmol $^{-1} \times$ second $^{-1}$ for gadoteridol. ${ }^{4,5}$ The presence of human serum albumin has no significant effect on the relaxivity of either agent because neither exhibits protein binding. The only significant difference between the agents is that gadobutrol is formulated at a $1.0-\mathrm{mol} / \mathrm{L}$ concentration while gadoteridol, similar to other GBCAs approved for CNS MR imaging, is formulated at a $0.5-\mathrm{mol} / \mathrm{L}$ concentration.

A previous single-center comparison of enhancement effectiveness between the 2 agents suggested the superiority of 1.0$\mathrm{mol} / \mathrm{L}$ gadobutrol over $0.5-\mathrm{mol} / \mathrm{L}_{\text {gadoteridol }}{ }^{6}$ in a limited study population (51 patients) with inconsistent, sequence-dependent differences in quantitative enhancement.

The purpose of this multicenter, multinational study was to determine, in a larger patient population by using a rigorous double-blind, randomized, intraindividual, crossover design, whether $1.0-\mathrm{mol} / \mathrm{L}$ gadobutrol has benefit over $0.5-\mathrm{mol} / \mathrm{L}$ gadoteridol for morphologic brain MR imaging when these agents are administered at identical $0.1-\mathrm{mmol} / \mathrm{kg}$ doses in 2 identical MR imaging examinations at $1.5 \mathrm{~T}$.

\section{MATERIALS AND METHODS}

The study was Health Insurance Portability and Accountability Act-compliant, was conducted according to good clinical practice standards, and was registered at www.clintrials.gov (reference NCT01613417). All patients signed an approved informed consent form before enrollment.

\section{Patients}

Two hundred twenty-nine patients referred for contrast-enhanced MR imaging for known or suspected brain tumors were enrolled in a consecutive manner at 19 participating centers between September 2012 and November 2013. The highest number of patients enrolled at any center was 30 . Thirteen centers enrolled between 9 and 25 patients. The remaining 5 centers enrolled between 1 and 4 patients. Patients were ineligible if they received any investigational drug within 30 days before study agent administration. Subjects were also excluded if they were to receive any treatment between the 2 examinations that could affect lesion visualization (eg, radiation therapy, steroids, or chemotherapy). Patients were also ineligible if they were pregnant or nursing or had impaired renal function, congestive heart failure, claustrophobia, gadolinium allergy, a cardiac pacemaker, or other contraindications to MR imaging.

The 229 enrolled patients (98 men, 131 women; mean age, $55.3 \pm 14.4$ years; range, $19-86$ years) were prospectively randomized to receive contrast agent according to 1 of 2 administration orders: Gadoteridol was administered first to patients in group A $(n=113)$, while gadobutrol was administered first to patients in group $\mathrm{B}(n=116)$.

\section{MR Imaging}

MR imaging was performed on $1.5 \mathrm{~T}$ systems from several vendors (Siemens Avanto $[n=26]$, Sonata $[n=10]$, Aera $[n=6]$, Symphony $[n=1]$, Siemens, Erlangen, Germany; Philips Achieva [ $n=101]$, Philips Healthcare, Best, the Netherlands; GE Signa Excite $[n=24]$, HDx $[n=45]$, Optima [ $n=16]$, GE Healthcare,
Milwaukee, Wisconsin) using a multichannel head coil. Although higher field strength $3 \mathrm{~T}$ systems are widely used, especially for their advantages for advanced imaging techniques, this study was performed at $1.5 \mathrm{~T}$ because these systems are still more commonly used throughout the United States, Europe, and other areas worldwide.

A rigorously controlled imaging protocol comprising T1weighted spin-echo (SE), T2-weighted fast spin-echo, and T2weighted FLAIR acquisitions before contrast injection and T1 SE and 3D-T1-weighted high-resolution gradient recalled-echo (GRE) acquisitions after injection ensured protocol uniformity across sites and within individual patients. Sequence parameters varied within predefined ranges necessitated by the use of different imaging systems. However, the same MR imaging scanner, imaging planes, section prescriptions, and sequence parameters were used for both examinations in each patient. Scan parameters were as follows - for the T1 SE sequence: $\mathrm{TR}=333-767 \mathrm{~ms}$, TE $=$ 7.7-16 ms, excitations $=1-3$, section thickness $=4-5 \mathrm{~mm}$, FOV $=17 \times 22-28 \times 28 \mathrm{~cm}$; for the T1 GRE sequence: TR $=$ $5.3-2050 \mathrm{~ms}$, TE $=1.19-7.24 \mathrm{~ms}$, flip angle $=8^{\circ}-30^{\circ}$, excitations $=0.8-1$, section thickness $=1-3 \mathrm{~mm}$, FOV $=23 \times 18-$ $28 \times 28 \mathrm{~cm}$. Parallel imaging was not used for any patient. Axial scans were all acquired along the inferior callosal plane to ensure image comparability among scans within patient comparisons and across patients.

IV contrast-agent administration was performed identically in both examinations at $0.1 \mathrm{mmol} / \mathrm{kg}$ of body weight $(0.2 \mathrm{~mL} / \mathrm{kg}$ for gadoteridol and $0.1 \mathrm{~mL} / \mathrm{kg}$ for gadobutrol) by using either a manual bolus injection $(n=207)$ or a power injector $(n=22)$. All injections were followed by a saline flush of up to $30 \mathrm{~mL}$. Each agent was administered by an independent drug-dispensing person in the order determined by a randomization list to maintain the study blinding.

Postcontrast image acquisition began at a prespecified time between 3 and 10 minutes after injection but could vary within this range, depending on the site-specific protocol. However, the timing and order of postcontrast sequences were mandated to be identical for both examinations within each patient. The interval between MR imaging examinations was $>48$ hours to avoid carryover effects but $<14$ days to minimize the chance of measurable lesion evolution.

Image Evaluation. All images were evaluated by 3 independent neuroradiologists (S.B., E.B., and T.H with 20, 11, and 24 years of neuroradiology experience, respectively) who were unaffiliated with the study centers and blinded to the contrast agent used, patient clinical and radiologic information, and interpretations by on-site investigators. Each reader evaluated all patient images separately and independently on a multimonitor workstation. The reading consisted of 2 sessions.

Diagnostic Performance. In the first session, each reader evaluated images presented in unpaired, randomized order to determine the extent of anatomic coverage (complete or partial) and to rate the overall quality of visualization (nondiagnostic, poor, fair, good, excellent). Any images rated nondiagnostic would be excluded from subsequent evaluation.

Next, assessments of diagnostic performance were performed 
by each offsite reader separately for lesions detected in each of the 2 examinations. For this assessment, each reader assigned a diagnosis to each detected lesion from a list of 99 possible diagnoses ratified by the World Health Organization ${ }^{7,8}$ that covers the range of nontumor diagnoses (white matter disease, vascular lesions, infective/inflammatory disease, infarct, hemorrhage, postoperative or posttreatment changes) and tumor diagnoses (astrocytic tumors, oligodendroglial tumors, ependymal cell tumors, mixed gliomas, neuroepithelial tumors of uncertain origin, tumors of the choroid plexus, neuronal and mixed neuronal-glial tumors, pineal parenchymal tumors, tumors with neuroblastic or glioblastic elements [embryonal tumors], tumors of the sellar region, hematopoietic tumors, germ cell tumors, tumors of the meninges, nonmeningothelial tumors of the meninges, tumors of cranial and spinal nerves, metastatic tumors, and cysts and tumorlike lesions).

For each lesion, readers assigned either a single diagnosis or could choose to assign differential diagnoses (ie, 2, 3, or $>3$ diagnoses). With this approach, a confidence score for correct lesion diagnosis was determined by using a 5-point scale as follows: 5 (single diagnosis, correctly matched with final TRUTH standard diagnosis); 4 (2 differential diagnoses, the first or second correctly matched); 3 (3 differential diagnoses; the first, second, or third correctly matched); 2 (>3 differential diagnoses; the first, second, or third correctly matched) or 1 (no match or nondiagnostic images or lesions confirmed at final diagnosis but not detected at MR imaging).

Subsequent comparisons of diagnostic performance (lesion detection rate, accuracy for tumor characterization - that is, the distinction between benign and malignant tumors based on World Health Organization brain tumor classification) and confidence for lesion characterization were performed for patients with histologically confirmed brain tumors after biopsy or surgical resection. For these evaluations, patients with only follow-up diagnostic data from alternative imaging procedures were excluded. A fourth independent neuroradiologist (T.F. with 20 years of neuroimaging experience), unaffiliated with the study centers and blinded to all clinical and radiologic information, matched the findings of the 3 blinded readers to the final lesion diagnosis for each patient.

Qualitative and Quantitative Assessment of Diagnostic Information. In the second reading session, qualitative and quantitative assessment of images from each patient was performed with images presented in global matched-pairs fashion. For each randomized patient number, all images from examination 1 were displayed simultaneously with the images from examination 2 . Each reader could perform all routine interactive image-manipulation functions (eg, window/level, zoom, pan) on both image sets. If the postinjection images from either examination were considered technically inadequate by any of the 3 readers (eg, if artifacts compromised interpretability), no further assessment was performed for that patient by that reader. Once the readers' assessments were recorded and signed off on an electronic Case Report Form, the database for that reading was automatically locked.

Qualitative Assessment. Technically adequate images were evaluated qualitatively for diagnostic information and scored in terms of the following: 1) overall diagnostic preference, 2) lesion border delineation, 3) disease extent, 4) visualization of lesion internal morphology, and 5) lesion contrast enhancement compared with surrounding normal tissue. All assessments were performed by using 3-point scales from -1 (examination 1 superior) through 0 (examinations equal) to +1 (examination 2 superior). For the various end points, superiority for 1 examination was recorded if it allowed better separation of $\geq 1$ lesion from surrounding tissue, structures, or edema; better definition of lesion extent; clearer depiction of intralesion features; better difference in signal intensity between lesions and surrounding normal tissue; or depiction of $\geq 1$ lesion seen only on that examination.

Quantitative Assessment. Quantitative evaluation was also performed by each reader, independently by using a simultaneous matched-pairs approach. Signal-intensity (SI) measurements were made by using ROIs positioned on areas of normal brain parenchyma and on up to 3 enhancing lesions per patient identified on postcontrast images from both examinations. Each region of interest placed on the selected postinjection image from one examination appeared simultaneously on the corresponding image from the other examination, to ensure that ROIs of equal size were positioned at identical coordinates on all corresponding image sets. Minor adjustments to ROI position were permitted to allow for slight differences in patient alignment, always taking care to avoid inclusion of vessels. When multiple lesions were present, ROIs were placed on up to 3 of the largest, most conspicuous lesions. A multimonitor imaging workstation (Aquarius, Version 4.4.1.4; TeraRecon, San Mateo, California) was used to determine SI values on a pixel-by-pixel basis and to calculate the percentage enhancement (E\%) of lesions and the lesion-to-background ratio (LBR) on T1 SE sequences by using the equations below:

$\mathrm{E} \%=\frac{\mathrm{SI} \text { of lesion }(\text { postcontrast })-\mathrm{SI} \text { of lesion (precontrast) }}{\text { SI of lesion (precontrast) }}$

$$
\mathrm{LBR}=\frac{\text { SI of lesion }}{\text { SI of brain }}
$$

\section{Safety Assessments}

Monitoring for adverse events was performed from the time the patient signed the informed consent form until 24 hours after administration of the first study agent and then from the moment the second study agent was administered until 24 hours after administration of the second agent. Events were classified as serious (death, life-threatening, requiring/prolonging hospitalization) or nonserious (mild [no disability/incapacity; self-resolving], moderate [no disability/incapacity; requiring treatment], or severe [temporary and/or mild disability/incapacity; requiring treatment]). Decisions on event severity and its relationship to the study agent (has reasonable possibility or not) were made by the investigating radiologist.

\section{Statistical Analysis}

Power determination was based on the primary efficacy assumption that a $0.1-\mathrm{mmol} / \mathrm{kg}$ dose of gadoteridol is noninferior to an equivalent dose of gadobutrol in terms of global diagnostic preference. Sample size was calculated (nQuery, Version 6.01; Statistical Solutions, Cork, Ireland) by using the Newcombe-Wilson 


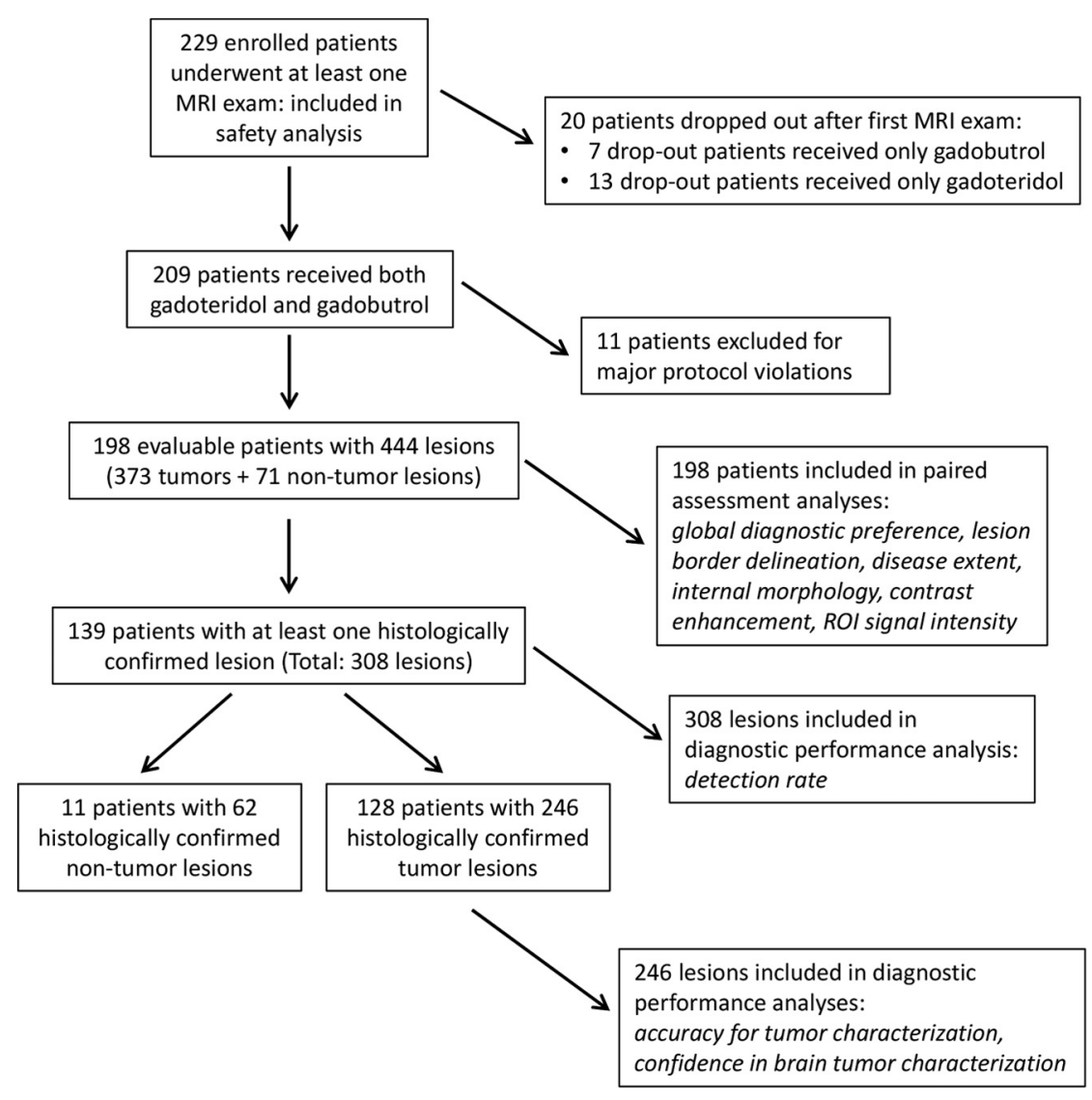

FIG 1. Flow chart outlining patient enrollment, drop-out rates, and lesion study populations. poor $(\kappa \leq 0.2)$. An overall mean confidence score \pm SD for lesion characterization was determined from the individual lesion confidence scores assigned to each patient. Comparison of mean confidence scores was performed by using a paired $t$ test. The Fisher exact test was used to compare the incidence of adverse events for the 2 agents and overall quality of visualization. All statistical tests were conducted at a significance level of $P<.05$.

\section{RESULTS}

\section{Patients}

All 229 enrolled patients underwent at least 1 contrast-enhanced MR imaging examination and were included in the overall safety population. Twenty patients discontinued after the first examination (13/113 [11.5\%] after gadoteridol; 7/116 [6.0\%] after gadobutrol; $P=.165)$. Reasons for discontinuation included withdrawal of consent $(n=11)$, surgical in tervention $(n=3)$, mild adverse event $(n=2)$, claustrophobia $(n=1)$, change of hospital $(n=1)$, inability to obtain intravenous access $(n=1)$, and lack of enhancing lesion $(n=1)$. Of the remaining $209(91.3 \%)$ patients, 11 were excluded from the efficacy population because of scoring method, which is based on the lower confidence limit for the difference in paired proportions; an estimated enrollment of 185 subjects was deemed necessary for the lower limit of the observed 2 -sided $95 \%$ confidence interval for the difference to exceed $-5 \%$ with $85 \%$ power. If one assumed a patient drop-out rate of $10 \%$, a minimum enrollment of 206 subjects was planned.

Analysis of blinded reader evaluations was performed by using the statistical software package SAS, Version 9.2 (SAS Institute, Cary, North Carolina). The distribution of reader preferences for the diagnostic information end points was tested by using the Wilcoxon signed rank test. The Altman general approximate normal method was used to estimate the 2 -sided $95 \%$ confidence interval for the difference in matched-paired proportions.

Differences in quantitative enhancement between gadoteridol and gadobutrol were analyzed by using a mixed-effects model. The change from predose was the response variable, and factors included in the model were patient, period, sequence, study agent, and predose score. Patient nested within sequence was the random effect.

Determinations of diagnostic performance, including 95\% confidence intervals, were performed for patients with histologically confirmed tumors from biopsy or surgery in terms of the lesion-detection rate and accuracy for tumor characterization. Comparison of detection rate and accuracy was performed by using the McNemar test. Interreader agreement was presented as percentage agreement and was assessed by using generalized $\kappa$ statistics. Agreement was classified as excellent $(\kappa>0.8)$, good $(\kappa=0.61-0.8)$, moderate $(\kappa=0.41-0.6)$, fair $(\kappa=0.21-0.4)$, or protocol violations (study agent doses missing or differing by $>15 \%, n=9$; differences of $>2$ minutes between injection and postdose acquisition start times, $n=2$ ). The final efficacy analysis population therefore comprised 198 patients, of which 93 (43 men, 50 women; mean age, $54.4 \pm 14.4$ years; range, $19-79$ ) were randomized to group $\mathrm{A}$, and 105 (47 men, 58 women; mean age, $55.9 \pm 14.3$ years; range, $25-82$ years), to group B (Fig 1 ). There were no significant between-group differences in sex $(P=.835)$, age $(P=.463)$, age groups $(18-64$ years, $\geq 65$ years; $P=.184)$, weight $(P=.071)$, height $(P=.503)$, or race $(P=.150)$ distribution.

Anatomic coverage was considered complete for all 198 patients by readers 1 and 3 and for 197/198 patients by reader 2 . All images from both agents were considered diagnostic by all readers, and no images were excluded due to motion degradation. Readers 1,2 , and 3 considered the overall quality of visualization to be good or excellent for $91.4 \%, 89.9 \%$, and $98.5 \%$ of patients, respectively, after gadoteridol, and for $92.9 \%, 90.4 \%$, and $100 \%$ of patients after gadobutrol with no significant differences noted ( $P=.709, P=1.0, P=.248$; readers 1,2 and 3, respectively). Four hundred forty-four lesions were identified on-site in these 198 patients (Table 1). Of these lesions, 373/444 (84\%) in 181 patients were diagnosed as tumors (293 [66\%] malignant; 80 [18\%] benign), while 71/444 (16\%) were nontumors. Among these 198 patients, 139 had at least 1 lesion that was confirmed histologically after biopsy or surgery. In these 139 patients there were 308 lesions (tumors and nontumors), which were included in subsequent analysis of the lesion-detection rate. Among these 139 pa- 
Table 1: TRUTH standard lesion diagnoses

\begin{tabular}{lr}
\hline \multicolumn{1}{c}{ Specific Diagnosis } & N $=\mathbf{4 4 4}(\%)$ \\
\hline Malignant tumor diagnoses ( $n=293)(66 \%)$ & \\
Anaplastic astrocytoma (grade III) & $12(2.7)$ \\
Glioblastoma multiforme (grade IV) & $55(12.4)$ \\
Anaplastic oligodendroglioma (grade III) & $4(0.9)$ \\
Anaplastic oligoastrocytoma (grade III) & $8(1.8)$ \\
Ependymoastrocytoma & $2(0.5)$ \\
Malignant lymphoma & $1(0.2)$ \\
Metastatic tumors & $211(47.5)$ \\
Benign tumor diagnoses ( $n=80)$ (18\%) & \\
Astrocytoma (grade II) & $2(0.5)$ \\
Pilocytic astrocytoma (noninvasive, grade I) & $1(0.2)$ \\
Oligodendroglioma (grade II) & $1(0.2)$ \\
Ependymoma (grade II) & $1(0.2)$ \\
Mixed oligoastrocytoma (grade II) & $1(0.2)$ \\
Pineocytoma (grade I) & $1(0.2)$ \\
Pituitary adenoma & $5(1.1)$ \\
Craniopharyngioma (grade I) & $1(0.2)$ \\
Meningioma (grade I) & $38(8.6)$ \\
Atypical meningioma (grade II) & $9(2.0)$ \\
Benign mesenchymal tumor & $1(0.2)$ \\
Melanocytoma & $1(0.2)$ \\
Hemangioblastoma (grade I) & $1(0.2)$ \\
Schwannoma (neurinoma, neurilemmoma) (grade I) & $16(3.6)$ \\
Cysts and tumorlike lesions (epidermoid) & $1(0.2)$ \\
Nontumor diagnoses ( $n=71)(16 \%)$ & \\
White matter disease & $1(0.2)$ \\
Vascular lesion & $10(2.3)$ \\
Infective/inflammatory disease & $2(0.5)$ \\
Infarct & $1(0.2)$ \\
Hemorrhage & $1(0.2)$ \\
Postoperative/posttreatment changes & $56(12.6)$ \\
\hline
\end{tabular}

tients, 128 had 246 lesions that were confirmed histologically as tumors, while the remaining 11 patients had lesions confirmed histologically as nontumors. All 246 histologically confirmed tumors were included in assessments of accuracy for tumor characterization (ie, benign-versus-malignant tumors based on World Health Organization brain tumor classification) and for confidence in brain tumor characterization (Fig 1).

\section{Qualitative Image Assessment}

Figure 2 graphically displays the results of the 3 blinded readers for global diagnostic preference, lesion-border delineation, disease extent, internal morphology, and qualitative assessment of contrast enhancement, respectively. No significant differences between gadoteridol and gadobutrol were noted by any reader for any parameter. The $95 \%$ confidence intervals for all qualitative assessments confirmed that gadoteridol is not inferior to gadobutrol. Agreement among the 3 blinded readers was high for all assessments, ranging from $82.5 \%$ of patients for assessment of lesion contrast enhancement to $97.9 \%$ of patients for definition of disease extent.

Examples of comparative enhancement between gadoteridol and gadobutrol are shown in Figs 3 and 4.

\section{Quantitative Evaluation}

Readers also recorded lesion signal-intensity measurements relative to normal brain parenchyma for up to 3 lesions in each patient. The mean percentage signal enhancement of lesions on T1 SE images was similar for gadoteridol and gadobutrol for all 3 readers (reader 1: $97.3 \%$ versus $96.9 \%[P=.620]$; reader $2: 95.6 \%$ versus $98.8 \%[P=.451]$; reader $3: 92.8 \%$ versus $95.3 \%[P=$ $.772])$.

No significant differences between gadoteridol and gadobutrol were noted by any reader for pre- to postdose changes in lesion-to-background ratio on T1 SE images (Fig 5). Similar findings were noted for assessments of T1 GRE images.

\section{Diagnostic Performance}

Lesion Detection. No significant differences between agents were noted by readers 1 and 2 in the number of patients with brain lesions, while only minimal differences were noted by reader 3 (Table 2). Similarly, no significant differences were noted by readers 1 and 2 in the number of lesions detected. On the basis of 308 lesions included in the analysis, all 3 readers agreed for $70.8 \%$ $(\kappa=0.39)$ of lesions after gadoteridol administration and for $72.4 \%(\kappa=0.47)$ of lesions after gadobutrol administration. At the patient level, on the basis of 139 subjects with histopathologic disease confirmation, all 3 readers agreed in $95.7 \%(\kappa=0.44)$ of patients after gadoteridol administration and in $95.0 \%(\kappa=0.48)$ of patients after gadobutrol.

Accuracy for Tumor Characterization. Readers 1 and 2 noted no significant differences between gadoteridol and gadobutrol for characterization of detected tumors either at the patient level or at the lesion level (Table 3). Conversely, improved lesion characterization with gadoteridol was noted by reader 3 . On the basis of 128 subjects with histologically confirmed brain tumors, all 3 readers agreed in their assessments for $70.3 \%$ $(\kappa=0.45)$ of patients after gadoteridol and for $66.4 \%(\kappa=$ $0.43)$ of patients after gadobutrol.

Confidence for Brain Tumor Diagnosis. Of 128 patients with histologically confirmed brain tumors, slightly higher mean confidence scores were assigned in the gadoteridol group (reader 1: $3.6 \pm 1.8$ versus $3.3 \pm 1.9, P=.016$; reader $2: 3.6 \pm 1.5$ versus $3.4 \pm 1.6, P=.011$; reader $3: 3.5 \pm 1.6$ versus $3.3 \pm 1.7, P=.119$ ), indicating more single diagnoses and fewer differential diagnoses with gadoteridol. Similar findings were noted for 246 histologically confirmed tumors (reader $1: 3.5 \pm 1.8$ versus $3.2 \pm 1.9, P=$ .001 ; reader $2: 3.7 \pm 1.6$ versus $3.4 \pm 1.7, P<.001$; reader $3: 3.3 \pm$ 1.7 versus $3.2 \pm 1.8, P=.033)$.

\section{Safety}

Fifteen $(6.8 \%)$ patients reported adverse events after gadoteridol administration. The most frequent events were dysgeusia $(n=4)$, nausea $(n=4)$, and headache $(n=2)$, followed by "feeling hot," lethargy, cough, dyspnea, pruritus, rash, dizziness, vomiting, migraine, urticaria, and vascular rupture. Two events in 2 patients were local injection site reactions. Eight (3.7\%) patients reported adverse events after gadobutrol administration. These included 1 case of injection site reaction plus dizziness, dysgeusia, headache, vomiting, paraesthesia, fatigue, dizziness, convulsion, and oropharyngeal pain. There was no significant difference in the incidence of adverse events between the 2 agents $(P=.199)$; no serious adverse events were reported for either agent. 
A
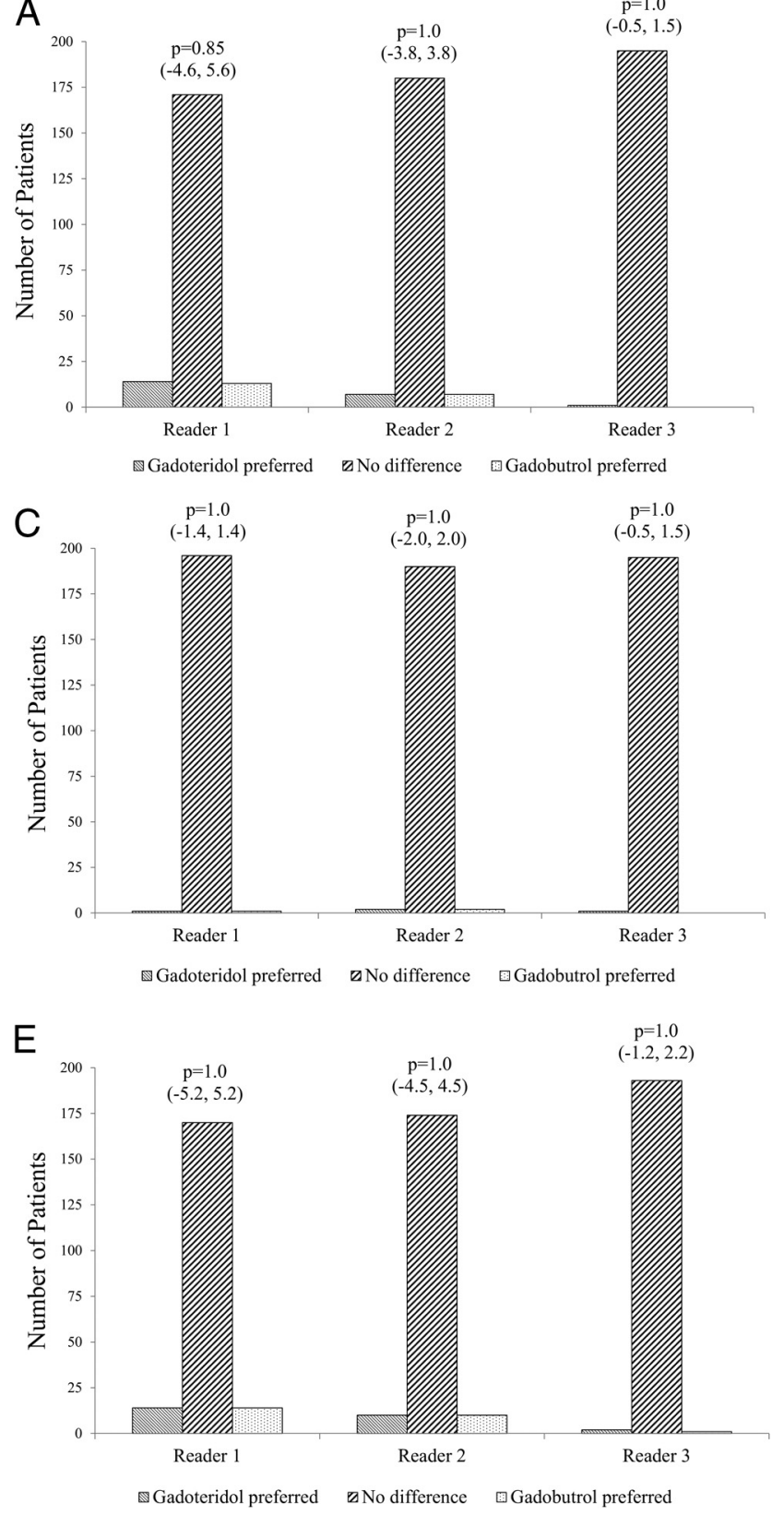

\section{DISCUSSION}

A previous small, single-center, intraindividual study compared contrast-enhancement effectiveness of $1.0 \mathrm{~mol} / \mathrm{L}$ gadobutrol and $0.5 \mathrm{~mol} / \mathrm{L}$ gadoteridol in 51 patients scheduled for neurosurgery. ${ }^{6}$ Independent injections of gadobutrol and gadoteridol at doses of $0.1-\mathrm{mmol} \mathrm{Gd} / \mathrm{kg}$ of body weight were administered in randomized order. For the primary efficacy variable "preference in contrast enhancement for one contrast agent or the other," the rate of "gadobutrol preferred" was significantly higher compared with the rate of "gadoteridol preferred."

Other studies have been performed as part of clinical trials for marketing approval for $1.0 \mathrm{~mol} / \mathrm{L}$ gadobutrol in $\mathrm{Japan}^{9}$ and the United States. ${ }^{10,11}$ The study in Japan was a multicenter, singleblind, randomized, intraindividual crossover comparison conducted in 175 patients. ${ }^{9}$ Each patient underwent 1 MR imaging examination with gadobutrol and the other with gadoteridol, each at a dose of $0.1 \mathrm{mmol} / \mathrm{kg}$ of body weight, administered twice,
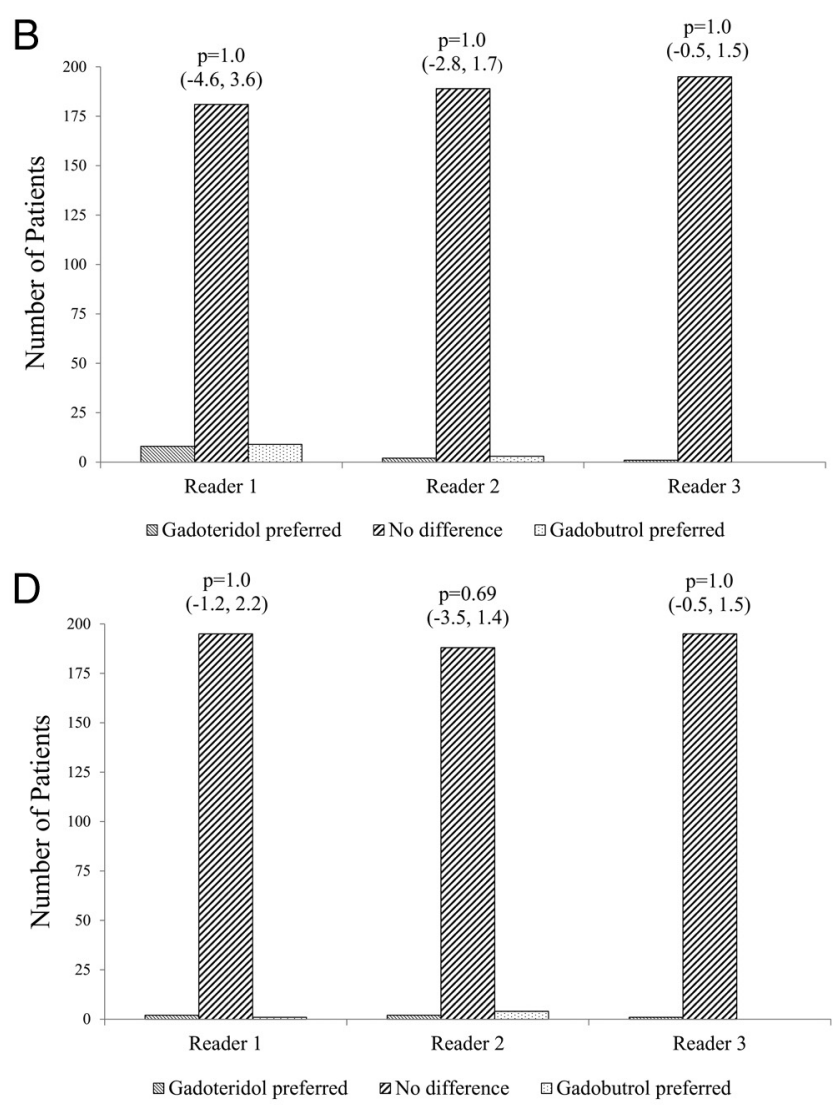

FIG 2. Bar graphs show reader preference and diagnostic results from 3 independent blinded readers for the following: global diagnostic preference $(A)$, border delineation $(B)$, internal morphology $(C)$, lesion extent $(D)$, and qualitative contrast enhancement $(E)$. Comparisons are based on 198 patients for reader 1, 194 patients for reader 2 and 196 patients for reader 3 . Each reader expressed no preference for either agent in the overwhelming number of cases for all 5 assessments. The small number of cases in which 1 agent is preferred is nearly equally distributed for gadobutrol versus gadoteridol. Note the very high reader agreement for all measures.

for a total dose of $0.2 \mathrm{mmol} / \mathrm{kg}$ of body weight. Imaging was performed after the first and second dose of gadobutrol, but only after the second dose of gadoteridol. Noninferiority of gadobutrol (both doses) to gadoteridol at $0.2 \mathrm{mmol} / \mathrm{kg}$ of body weight was demonstrated. However, a major limitation of that study is that though a single $0.1-\mathrm{mmol} / \mathrm{kg}$ dose of gadoteridol was administered, no images were acquired and the 2 agents were not compared at a single dose. Hence, it is not possible to determine whether a single dose of gadoteridol would have proved noninferior to a single dose of gadobutrol. The second phase III clinical trial, which is unpublished, found no differences between a $0.1-\mathrm{mmol} / \mathrm{kg}$ dose of gadobutrol and an equivalent $0.1-\mathrm{mmol} / \mathrm{kg}$ dose of gadoteridol for enhanced CNS MR imaging. ${ }^{1,10,11}$ In this study, no differences were noted in qualitative visualization end points (contrast enhancement, lesion border delineation, and visualization of lesion internal morphology), number of lesions detected, or accuracy for lesion 



FIG 3. A 61-year-old man with brain metastases from primary lung cancer. Images were acquired before ( $A$, unenhanced TT SE) and after ( $B$, TI SE; $C$, high-resolution TI GRE) administration of gadoteridol and before $(D$, unenhanced TI SE) and after ( $E$, TT SE; $F$, high-resolution $T 7$ GRE) administration of gadobutrol. Two lesions clearly seen in both examinations show no differences in contrast enhancement or in the size of lesions.

diagnosis. The results of the study are reflected in the current prescribing information for gadobutrol in the United States. ${ }^{1}$

Our findings confirm that no major differences between gadoteridol and gadobutrol are apparent when single $0.1-\mathrm{mmol} / \mathrm{kg}$ doses of each agent are administered to patients with confirmed brain lesions. Specifically, evaluating images in matched pairs from $\geq 194$ patients for qualitative visualization end points, 3 blinded, expert neuroradiologists expressed no preference for either agent in most cases. In the few cases in which a reader expressed preference, the number of preferences for gadobutrol was approximately equal to the number of preferences for gadoteridol. Similar findings were noted for quantitative enhancement measurements: No significant differences between gadobutrol and gadoteridol were noted either for mean percentage signal enhancement or for pre- to postdose changes in the lesion-tobackground ratio. The interreader agreement for assessment of images was high in all cases (complete agreement for $82.5 \%-$ $97.9 \%$ of patients among qualitative end points). For patients with histologically confirmed brain tumors, no significant differences were noted by 2 blinded readers for either lesion detection at
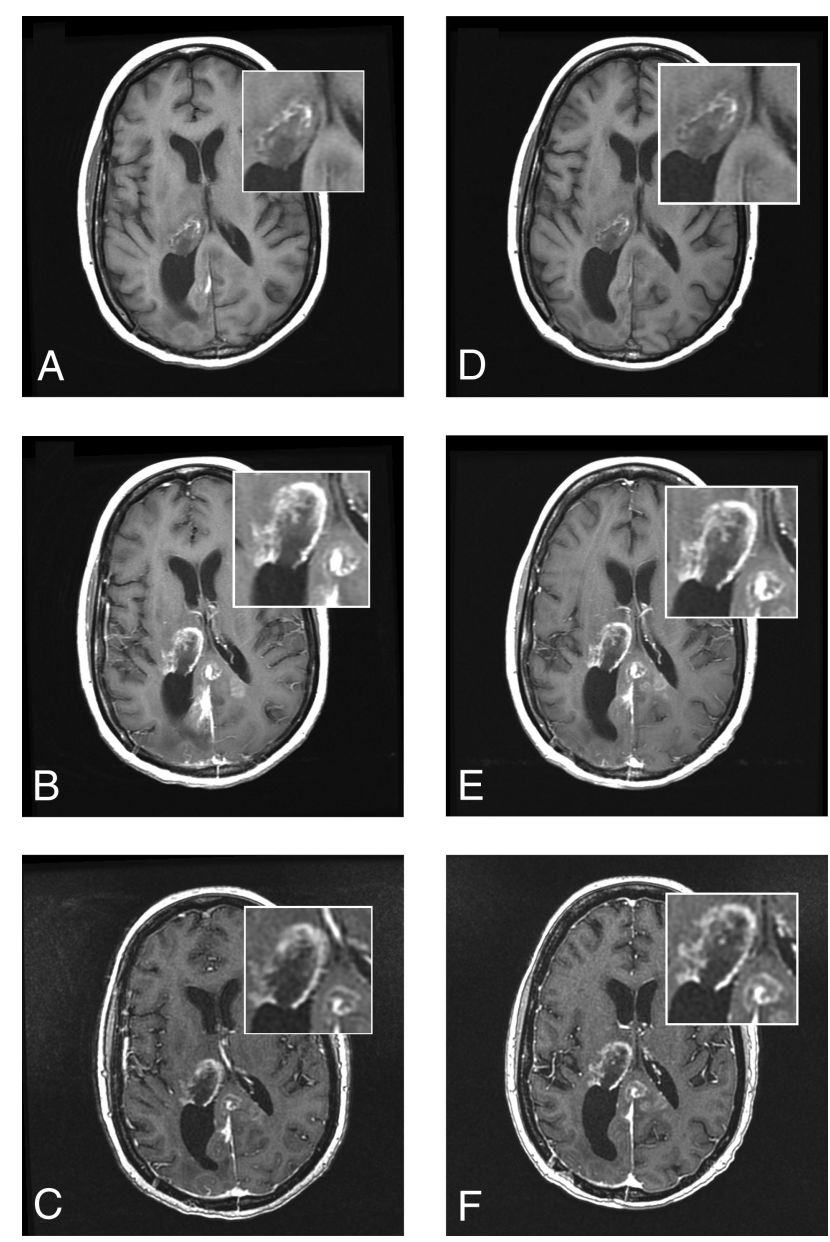

FIG 4. A 51-year-old woman with glioblastoma multiforme. Images were acquired before $(A$, unenhanced $\mathrm{TI} \mathrm{SE})$ and after $(B, \mathrm{~T} \mathrm{SE} ; C$, high-resolution TI GRE) administration of gadoteridol and before ( $D$, unenhanced TI SE) and after ( $E$, TI SE; $F$, high-resolution TI GRE) administration of gadobutrol. A rim-enhancing mass in the right thalamus with extension into the posterior interhemispheric region is clearly seen in both examinations. No differences in contrast enhancement or in the size of lesions are apparent.

both patient and lesion levels or for characterization of tumors as malignant or benign. Although 1 blinded reader noted a significant benefit for gadoteridol in patients with brain tumors, the conclusion of our study is that the preference and diagnostic performance of gadobutrol and gadoteridol are similar for imaging of brain tumors when administered at an approved dose of 0.1 $\mathrm{mmol} / \mathrm{kg}$ of body weight.

Although the interreader agreement for lesion characterization was considered moderate $(\kappa=0.43-0.45)$, evaluations were performed in a fully blinded fashion (ie, without access to patient clinical history or the results of other diagnostic tests), and only images acquired by using conventional sequences were evaluated (ie, without the additional information available from advanced imaging techniques such as perfusion, diffusion, or spectroscopic imaging). Moreover, at variance with many literature reports, agreement in this study was determined across 3 readers rather than 2 . Hence, if 2 of the 3 readers agreed in their diagnosis but the third disagreed (eg, regarding classification of a detected glioma as grade III [malignant] versus grade II [benign] according to World Health Organization criteria $^{7,8}$ ), the overall consensus was dis- 
agreement among readers. In this context, overall agreement across 3 readers for $66.4 \%$ of patients $(\kappa=0.43)$ after gadobutrol and $70.3 \%$ of patients $(\kappa=0.45)$ after gadoteridol was good.

Two conclusions can be derived from this study. First, findings demonstrate that the 2-fold higher concentration of gadobutrol in the commercially available formulation provides no advantage for morphologic imaging of brain lesions. This conclusion is not unexpected, given that image acquisition for morphologic imaging of brain tumors typically begins at least 3-5 minutes following contrast administration, by which time contrast equilibration will have occurred, which would obviate any potential benefits of a higher administered concentration. ${ }^{12,13}$ Second, although $r 1$ relaxivity is a major factor for contrast efficacy, ${ }^{13}$ the slightly different $r 1$ relaxivity values for gadobutrol and gadoteridol are insufficient to show any discernable clinical effect, either for qualitative or quantitative lesion enhancement or for diagnostic performance.

These conclusions are supported by other intraindividual crossover studies performed at $1.5 \mathrm{~T}$ comparing these agents with other GBCAs for brain tumor imaging. Thus, Greco et al ${ }^{14}$ com-



FIG 5. Blinded reader comparison of mean postcontrast-precontrast lesion-to-background ratio on T1 SE sequences after $0.1-\mathrm{mmol} / \mathrm{kg}$ doses of gadoteridol and gadobutrol. No significant differences were noted by any reader.

Table 2: Detection of histologically confirmed brain tumors on MR images acquired after administration of $0.1-\mathrm{mmol} / \mathrm{kg}$ gadoteridol or $0.1-\mathrm{mmol} / \mathbf{k g}$ gadobutrol in 139 patients with 308 brain lesions subsequently confirmed at biopsy or surgery

\begin{tabular}{|c|c|c|c|c|c|c|}
\hline \multirow[b]{2}{*}{ Lesion Detection } & \multicolumn{2}{|c|}{ Reader 1} & \multicolumn{2}{|c|}{ Reader 2} & \multicolumn{2}{|c|}{ Reader 3} \\
\hline & Gadoteridol & Gadobutrol & Gadoteridol & Gadobutrol & Gadoteridol & Gadobutrol \\
\hline No. patients with tumors detected at MRI & $133(95.7 \%)$ & $135(97.1 \%)$ & $137(98.6 \%)$ & $136(97.8 \%)$ & $136(97.8 \%)$ & $132(95.0 \%)$ \\
\hline$P$ value (95\% $\mathrm{Cl}$ of difference, \%) & \multicolumn{2}{|c|}{$.317(-4.2$ to 1.4$)$} & \multicolumn{2}{|c|}{$.564(-1.7$ to 3.2$)$} & \multicolumn{2}{|c|}{.046 (0.1 to 5.7$)$} \\
\hline No. tumors detected at MRI & 240 (77.9\%) & $236(76.6 \%)$ & 269 (87.3\%) & $263(85.4 \%)$ & 230 (74.7\%) & 220 (71.4\%) \\
\hline$P$ value $(95 \% \mathrm{Cl}$ of difference, $\%)$ & \multicolumn{2}{|c|}{0.480 ( -2.3 to 4.9$)$} & \multicolumn{2}{|c|}{$0.239(-1.3$ to 5.2$)$} & \multicolumn{2}{|c|}{0.018 (0.6 to 5.9$)$} \\
\hline
\end{tabular}

Table 3: Accuracy for brain tumor characterization on MR images acquired after administration of $0.1-\mathrm{mmol} / \mathbf{k g}$ gadoteridol or $0.1-\mathrm{mmol} / \mathrm{kg}$ gadobutrol in 128 patients with 246 histologically confirmed brain tumors

\begin{tabular}{|c|c|c|c|c|c|c|}
\hline \multirow[b]{2}{*}{ Lesion Characterization } & \multicolumn{2}{|c|}{ Reader 1} & \multicolumn{2}{|c|}{ Reader 2} & \multicolumn{2}{|c|}{ Reader 3} \\
\hline & Gadoteridol & Gadobutrol & Gadoteridol & Gadobutrol & Gadoteridol & Gadobutrol \\
\hline No. patients with tumors correctly characterized at MRI & $94(73.4 \%)$ & $96(75.0 \%)$ & $106(82.8 \%)$ & $101(78.9 \%)$ & $93(72.7 \%)$ & $83(64.8 \%)$ \\
\hline$P$ value (95\% Cl of difference, \%) & \multicolumn{2}{|c|}{$.695(-9.4$ to 6.2$)$} & \multicolumn{2}{|c|}{$.132(-1.1$ to 8.9$)$} & \multicolumn{2}{|c|}{$.012(1.8$ to 13.8$)$} \\
\hline No. tumors correctly characterized at MRI & $169(68.7 \%)$ & $164(66.7 \%)$ & $198(80.5 \%)$ & $188(76.4 \%)$ & $166(67.5 \%)$ & $148(60.2 \%)$ \\
\hline$P$ value $(95 \% \mathrm{Cl}$ of difference, $\%)$ & \multicolumn{2}{|c|}{$.492(-3.8$ to 7.8$)$} & \multicolumn{2}{|c|}{$.059(-0.1$ to 8.3$)$} & \multicolumn{2}{|c|}{$.001(3.0$ to 11.6$)$} \\
\hline
\end{tabular}

enhancement following gadobutrol was approximately $9 \%$ higher than that following gadoterate meglumine, but this yielded no significant difference between the 2 agents for measured contrast-tonoise ratio, and no differences in the number of lesions detected with either agent were observed. In comparison, in the latter study, ${ }^{16}$ all 3 blinded readers demonstrated a highly significant $(P<$ $.0001)$ preference for the higher relaxivity $0.5-\mathrm{mol} / \mathrm{L}$ gadobenate dimeglumine over $1.0-\mathrm{mol} / \mathrm{L}$ gadobutrol for all qualitative end points (lesion-border delineation, definition of disease extent, visualization of lesion internal morphology, lesion contrast enhancement, and global diagnostic preference) with good interreader agreement for all evaluations. In addition, significant superiority was noted for all quantitative assessments with a mean dif- 
ference of approximately $22 \%$ in the percentage lesion enhancement between gadobenate dimeglumine and gadobutrol.

Together with our findings, these studies suggest that only $r 1$ differences of a certain magnitude are sufficient to elicit discernable clinical differences in GBCA performance. Thus, intraindividual studies that have compared GBCAs with similar $r 1$ relaxivity have generally demonstrated similar image quality and diagnostic performance, ${ }^{10,11,14}$ while studies that have compared GBCAs with higher $r 1$ have demonstrated measurable differences in image preference and diagnostic performance. ${ }^{16}$

In terms of safety, both gadoteridol and gadobutrol, given at a dose of $0.1 \mathrm{mmol} / \mathrm{kg}$ of body weight, were safe and well-tolerated in patients with suspected or confirmed brain pathology, with no serious adverse events reported for either agent. The rates of adverse events were similar for the 2 agents and were similar to rates reported previously for the 2 agents in other comparative studies; in the phase II/III study performed in Japan, 8 adverse events in 7 $(4.3 \%)$ patients were reported following cumulative doses of 0.2 $\mathrm{mmol} / \mathrm{kg}$ of body weight of both gadobutrol and gadoteridol, ${ }^{9}$ while in a smaller intraindividual comparative study, ${ }^{6}$ neither agent caused any unexpected or serious adverse events during the study period.

The principal limitations of our study are that although 198 of 229 enrolled patients were included in the per-protocol efficacy population, histologic confirmation of disease was available for only 139 patients, of which only 128 were diagnosed as having brain tumors. On the other hand, this population size and the number of confirmed tumors evaluated $(n=246)$ are comparable with previous intraindividual comparisons of GBCAs for brain tumor imaging ${ }^{14-16}$ and should be sufficiently robust to provide a true reflection of the comparable performance of these 2 GBCAs. Additionally, a second limitation is that advanced imaging techniques (eg, perfusion, diffusion, and functional techniques) were not performed. Such techniques are often used to improve the grading and characterization of brain tumors and might have improved both diagnostic performance and interreader agreement in this study had they been used. Although previous studies have compared GBCAs for perfusion imaging, ${ }^{13}$ future studies might benefit from including this technique in the imaging protocol.

In summary, the overriding conclusion of our study is that gadoteridol and gadobutrol confer similar image enhancement and diagnostic performance when administered under identical conditions at an approved dose of $0.1 \mathrm{mmol} / \mathrm{kg}$ of body weight. The similar performance of these 2 GBCAs shows that the small difference in $r 1$ values between the 2 agents does not confer any diagnostic advantage. Furthermore, our study confirms the findings of a previous multicenter study ${ }^{10,11}$ showing that the 2 -fold higher gadolinium concentration of the gadobutrol formulation has no significant impact on routine morphologic imaging of brain lesions.

\section{CONCLUSIONS}

Gadoteridol and gadobutrol at $0.1 \mathrm{mmol} / \mathrm{kg}$ of body weight provide similar qualitative and quantitative imaging results for visualization and diagnosis of brain lesions without any significant differences in safety or tolerability.

\section{ACKNOWLEDGMENTS}

The authors thank Sally Friend (University of Washington Medical Center, Seattle, Washington), Tomasz Dróżdż (Świętokrzyskie Centrum Onkologii, Kielce, Poland), Matthew White (Nebraska Medical Center, Omaha, Nebraska), Armando Tartaro (Università degli Studi “G. D’Annunzio,” Chieti, Italy), Jacqueline Brunetti (Holy Name Hospital, Teaneck, New Jersey), Barbara Paraniak-Gieszczyk (Helimed Diagnostic Imaging, Katowice, Poland), Linda Anderson (Seaman Family MR Research Centre, University of Calgary, Alberta, Canada), Simona Gaudino (Policlinico “Agostino Gemelli," Rome, Italy), and Daniel S. Gurell (University Diagnostic Medical Imaging PC, Bronx, New York) for enrolling patients into the study, and James R. Fink, MD (University of Washington Medical Center, Seattle, Washington) for assistance with recruiting and image scoring. In addition, the authors are indebted to Ningyan Shen, MD, PhD (Bracco Diagnostics, Monroe, New Jersey) for additional statistical analyses performed for this study.

Disclosures: Kenneth R. Maravilla—RELATED: Grant: Bracco Diagnostics, ${ }^{*}$ Comments: funding support for clinical trial study; Support for Travel to Meetings for the Study or Other Purposes: Bracco Diagnostics, Comments: travel support to attend 1-day Investigator meeting prior to start of trial; Provision of Writing Assistance, Medicines, Equipment, or Administrative Support: Bracco Diagnostics, Comments: no monetary value, only statistical support; UNRELATED: Consulting Fee or Honorarium: Bracco, Guerbet; Grants/Grants Pending: Bayer, Bracco, Guerbet; Payment for Development of Educational Presentations: Bracco. Gianpaolo PirovanoRELATED: Employment: Bracco Diagnostics, Comments: employee of trial-sponsoring company. Miles A. Kirchin—RELATED: Employment: Bracco Diagnostics, Comments: employee of trial-sponsoring company. Toshinori Hirai-RELATED: Consulting Fee or Honorarium: Bracco Diagnostics; Support for Travel to Meetings for the Study or Other Purposes: Bracco Diagnostics. Martin P. Smith-RELATED: Grant: Bracco Diagnostics, ${ }^{*}$ Comments: My institution was paid by Bracco for each patient recruited into this study in order to screen them, complete the required documents, perform the MRIs, and evaluate the images; UNRELATED: Grants/Grants Pending: Bracco Diagnostics, ${ }^{*}$ Comments: paid for recruiting patients for another gadoliniumbased contrast agent study; Other: Bayer Pharmaceuticals, Comments: paid for recruiting patients for a gadolinium-based contrast agent study. Mayank GoyalUNRELATED: Consulting Fee or Honorarium: Covidien, Comments: for teaching engagements; Grants/Grants Pending: Covidien, ${ }^{\star}$ Comments: partial funding of the ESCAPE trial; Payment for Lectures Including Service on Speakers Bureaus: Covidien, Comments: for lecturing on acute stroke. Joseph J. Baima-RELATED: Grant: Bracco Diagnostics, ${ }^{*}$ Comments: Principal Investigator in the Bracco Study PH 107. Robert Babbel—RELATED: Grant: Samaritan Health Services.* Jan Žižka—RELATED: Consulting Fee or Honorarium: University Hospital Hradec Kralove.* Asim K. Bag-UNRELATED: Consulting Fee or Honorarium: Guerbet, Comments: Consultant to Dotarem; Advisory Board. Stefano Bastianello-RELATED: Consulting Fee or Honorarium: Bracco Diagnostics, Comments: for evaluating MR imaging. Eva Bueltmann—RELATED: Consulting Fee or Honorarium: Bracco Diagnostics, Comments: honorarium for blinded reading. Małgorzata Urbańczyk-Zawadzka—UNRELATED: Payment for Manuscript Preparation: PZWL; Grants/Grants Pending: John Paul II Hospital, Kraków, Poland. *Money paid to the institution.

\section{REFERENCES}

1. Gadavist product label. http://www.accessdata.fda.gov/drugsatfda_ docs/label/2011/201277s000lbl.pdf. Accessed April 11, 2014

2. ProHance product label. http://www.accessdata.fda.gov/drugsatfda docs/label/2010/020131s024lbl.pdf. Accessed April 11, 2014

3. Hao D, Ai T, Goerner F, et al. MRI contrast agents: basic chemistry and safety. J Magn Reson Imaging 2012;36:1060-71

4. Rohrer M, Bauer H, Mintorovitch J, et al. Comparison of magnetic properties of MRI contrast media solutions at different magnetic field strengths. Invest Radiol 2005;40:715-24

5. Pintaske J, Martirosian P, Graf H, et al. Relaxivity of gadopentetate dimeglumine (Magnevist), gadobutrol (Gadovist), and gadobenate dimeglumine (MultiHance) in human blood plasma at $0.2,1.5$, and 3 Tesla. Invest Radiol 2006;41:213-21 
6. Koenig M, Schulte-Altedorneburg G, Piontek M, et al. Intra-individual, randomised comparison of the MRI contrast agents gadobutrol versus gadoteridol in patients with primary and secondary brain tumours, evaluated in a blinded read. Eur Radiol 2013; 23:3287-95

7. Lopes MB, VandenBerg SR, Scheithauer BW. The World Health Organization classification of nervous system tumors in experimental neuro-oncology. In: Levine AJ, Schmidek HH, eds. Molecular Genetics of Nervous System Tumors. New York: Wiley-Liss; 1993:1-36

8. Kleihues P, Burger PC, Scheithauer BW. The new WHO classification of brain tumours. Brain Pathol 1993;3:255-68

9. Katakami N, Inaba Y, Sugata S, et al. Magnetic resonance evaluation of brain metastases from systemic malignances with two doses of gadobutrol 1.0M compared with gadoteridol: a multicenter, phase II/III study in patients with known or suspected brain metastases. Invest Radiol 2011;46:411-18

10. Peripheral and Central Nervous System Drugs Advisory Committee. FDA Advisory Committee Briefing Document. New Drug Application 201-277. http://www.fda.gov/downloads/AdvisoryCommittees/ CommitteesMeetingMaterials/Drugs/PeripheralandCentralNervous SystemDrugsAdvisoryCommittee/UCM240354.pdf. Accessed May 8, 2014

11. Center for Drug Evaluation and Research. Statistical Review and Evaluation. Gadovist. http://www.fda.gov/downloads/Advisory
Committees/CommitteesMeetingMaterials/Drugs/Peripheraland CentralNervousSystemDrugsAdvisoryCommittee/UCM255169.pdf. Accessed May 8, 2014

12. Heiland S, Erb G, Ziegler S, Krix M. Where contrast agent concentration really matters: a comparison of CT and MRI. Invest Radiol 2010;45:529-37

13. Kanal E, Maravilla K, Rowley HA. Gadolinium contrast agents for CNS imaging: current concepts and clinical evidence. AJNR Am J Neuroradiol 2014;35:2215-26

14. Greco A, Parker JR, Ratcliffe CG, et al. Phase III, randomized, double blind, crossover comparison of gadoteridol and gadopentetate dimeglumine in magnetic resonance imaging of patients with intracranial lesions. Australas Radiol 2001;45:457-63

15. Anzalone N, Scarabino T, Venturi C, et al. Cerebral neoplastic enhancing lesions: multicenter, randomized, crossover intraindividual comparison between gadobutrol $(1.0 \mathrm{M})$ and gadoterate meglumine $(0.5 \mathrm{M})$ at $0.1 \mathrm{mmolGd} / \mathrm{kg}$ body weight in a clinical setting. Eur J Radiol 2013;82:139-45

16. Seidl Z, Vymazal J, Mechl M, et al. Does higher gadolinium concentration play a role in the morphologic assessment of brain tumors? Results of a multicenter intraindividual crossover comparison of gadobutrol versus gadobenate dimeglumine (the MERIT Study). AJNR Am J Neuroradiol 2012;33:1050-58 